\title{
UNIVALENT HARMONIC FUNCTIONS GENERATED BY RUSCHEWEYH DERIVATIVES OF ANALYTIC FUNCTIONS
}

\author{
Om P. Ahuja, Subzar Beig and V. Ravichandran
}

Abstract. For $\lambda \geq 0, p>0$ and a normalized univalent function $f$ defined on the unit disk $\mathbb{D}$, we consider the harmonic function defined by

$$
T_{\lambda, p}[f](z)=\frac{\mathcal{D}^{\lambda} f(z)+p z\left(\mathcal{D}^{\lambda} f(z)\right)^{\prime}}{p+1}+\frac{\overline{\mathcal{D}^{\lambda} f(z)-p z\left(\mathcal{D}^{\lambda} f(z)\right)^{\prime}}}{p+1}, \quad z \in \mathbb{D},
$$

where the operator $\mathcal{D}^{\lambda}$ is the familiar $\lambda$-Ruscheweyh derivative operator. We find some necessary and sufficient conditions for the univalence, starlikeness and convexity as well as the growth estimate of the function $T_{\lambda, p}[f]$. An extension of the above operator is also given.

2010 Mathematics Subject Classification: 30C45.

Keywords: harmonic mappings; Ruscheweyh derivative; univalence; starlikeness; convexity; convexity in one direction

\section{INTRODUCTION}

Let $\mathcal{H}$ denote the class of complex-valued harmonic functions $f=h+\bar{g}$ defined in the unit disk $\mathbb{D}=\{z \in \mathbb{C}:|z|<1\}$, where $h$ and $g$ are analytic functions given by

$$
h(z)=z+\sum_{m=2}^{\infty} a_{m} z^{m}, \quad g(z)=\sum_{m=1}^{\infty} b_{m} z^{m} .
$$

By Lewy's Theorem [9], the function $f=h+\bar{g} \in \mathcal{H}$ is sense-preserving if and only if the Jacobian $J_{f}(z)=\left|h^{\prime}(z)\right|^{2}-\left|g^{\prime}(z)\right|^{2}$ is positive, or equivalently $\left|g^{\prime}\right|<\left|h^{\prime}\right|$ in $\mathbb{D}$. Let $\mathcal{S}_{H}$ be the subclass of $\mathcal{H}$ consisting of univalent and sense-preserving functions. A domain is said to be convex in the direction of real (or imaginary) axis if every line parallel to the real (or imaginary) axis has a connected intersection with the domain. The following theorem due to Clunie and Sheil-Small [5] and Sheil-Small [16] gives a technique of constructing univalent harmonic mappings in a given direction, known as "Shearing Method." 
Om P. Ahuja, Subzar Beig and V. Ravichandran - Univalent Harmonic Functions

Theorem 1. Let the function $f=h+\bar{g}$ be harmonic and locally univalent function in $\mathbb{D}$. Then

(1) the function $F=h-g \in \mathcal{S}$ and $F(\mathbb{D})$ is convex in the direction of real axis $\Longleftrightarrow$ the function $f=h+\bar{g}$ is univalent and convex in the direction of real axis.

(2) the function $F=h+g \in \mathcal{S}$ and $F(\mathbb{D})$ is convex in the direction of imaginary axis $\Longleftrightarrow$ the function $f=h+\bar{g}$ is univalent and convex in the direction of imaginary axis.

Using Theorem 1, Clunie and Sheil-Small [5] proved that if the functions $H_{0}$ and $G_{0}$ are analytic in $\mathbb{D}$ with $H_{0}(z)+G_{0}(z)=z /(1-z)$ and $G_{0}^{\prime}(z) / H_{0}^{\prime}(z)=-z$, then the resulting harmonic function $T_{0}:=H_{0}+\bar{G}_{0}$ is univalent and maps $\mathbb{D}$ onto the right half-plane $\{w \in \mathbb{C}: \operatorname{Re} w>-1 / 2\}$. In fact,

$$
T_{0}(z)=\frac{1}{2}\left(\frac{z}{1-z}+\frac{z}{(1-z)^{2}}\right)+\frac{1}{2} \overline{\left(\frac{z}{1-z}-\frac{z}{(1-z)^{2}}\right)}
$$

which may be expressed as

$$
T_{0}(z)=\frac{1}{2}\left(I(z)+z I^{\prime}(z)\right)+\frac{1}{2} \overline{\left(I(z)-z I^{\prime}(z)\right)}
$$

where

$$
I(z)=\frac{z}{1-z}
$$

is the analytic right-half plane mapping. The function $T_{0}$ is well-known in the theory of univalent harmonic functions and it acts extremal for many harmonic inequalities concerning the subclass of $\mathcal{S}_{H}$ consisting of convex functions.

Let $\mathcal{A}$ denote the class of all analytic functions $f$ defined in $\mathbb{D}$ normalized by $f(0)=0=f^{\prime}(0)-1$ and suppose that $\mathcal{S}$ is its subclass consisting of univalent functions. Motivated by the description of the right half-plane mapping $T_{0}$, we define a differential operator which is closely related to Ruscheweyh derivatives. If $f \in \mathcal{A}$, then for each $\lambda \geq 0$ and $p>0$, we define

$$
T_{\lambda, p}[f](z)=\frac{\mathcal{D}^{\lambda} f(z)+p z\left(\mathcal{D}^{\lambda} f(z)\right)^{\prime}}{p+1}+\frac{\overline{\mathcal{D}^{\lambda} f(z)-p z\left(\mathcal{D}^{\lambda} f(z)\right)^{\prime}}}{p+1}, \quad z \in \mathbb{D},
$$

where the operator $\mathcal{D}^{\lambda}: \mathcal{A} \rightarrow \mathcal{A}$ is $\lambda$-Ruscheweyh derivative of

$$
f(z)=z+\sum_{m=2}^{\infty} a_{m} z^{m}
$$


Om P. Ahuja, Subzar Beig and V. Ravichandran - Univalent Harmonic Functions

given by

$$
\mathcal{D}^{\lambda} f(z)=\frac{z}{(1-z)^{\lambda+1}} * f(z)=z+\sum_{m=2}^{\infty} \frac{(\lambda+1)_{m-1}}{(m-1) !} a_{m} z^{m}
$$

where

$$
(\lambda+1)_{m-1}=(\lambda+1)(\lambda+2) \cdots(\lambda+m-1) .
$$

Here $*$ is the convolution (or Hadamard product) of two power series of given two functions. For properties of Ruscheweyh derivatives, one may refer to $[1,2,13]$. The following example justify the need of the operator defined by (2).

Example 1. The operator $T_{0}(z)=T_{0,1}[I]$ was introduced and studied by Clunie and Sheil-Small[5]. In 2008, Muir [10] proved that $T_{p}[I]=T_{0, p}[I]$ is a harmonic right half-plane mapping of $\mathbb{D}$ onto the right half-plane $\{w \in \mathbb{C}: \operatorname{Re} w>-1 /(1+p)\}$ for each $p>0$. The operator $T_{p}[f]=T_{0, p}[f]$ for $f \in \mathcal{S}$ and $p>0$ was studied by Muir [11]. In [15], Ruscheweyh and Suffridge defined continuous extension of the de la Vallee Poussin means $\mathcal{V}_{\mu}: \mathbb{D} \longrightarrow \mathbb{C}$, by

$$
\mathcal{V}_{\mu}(z)=\frac{\mu z}{\mu+1}{ }_{2} \mathcal{F}_{1}(1,1-\mu, 2+\mu,-z), \quad \mu>0
$$

where ${ }_{2} \mathcal{F}_{1}$ is the Gaussian hypergeometric function. These authors also proved that the mapping

$$
T_{p}\left[\mathcal{V}_{\mu}\right]=\frac{\mathcal{V}_{\mu}+p z \mathcal{V}_{\mu}^{\prime}}{p+1}+\frac{\overline{\mathcal{V}_{\mu}-p z \mathcal{V}_{\mu}^{\prime}}}{p+1}
$$

is a harmonic mapping of $\mathbb{D}$ onto a convex domain for each $\mu \geq \frac{1}{2}$ and $p \geq 0$.

\section{PRELIMINARIES}

Let $\mathcal{R}_{\lambda}(\alpha)$ denote the class of functions $f \in \mathcal{A}$ satisfying the condition

$$
\operatorname{Re}\left(\frac{z\left(\mathcal{D}^{\lambda} f(z)\right)^{\prime}}{\mathcal{D}^{\lambda} f(z)}\right)>\alpha
$$

for some $\lambda>-1, \alpha<1$, and for all $z \in \mathbb{D}$, where $\mathcal{D}^{\lambda}: \mathcal{A} \rightarrow \mathcal{A}$ is the $\lambda$-Ruscheweyh derivative operator defined by (4). The class $\mathcal{R}_{\lambda}(\alpha)$ was introduced and studied by first author in $[1,2]$. In particular, note that $\mathcal{R}_{0}(\alpha)=\mathcal{S}^{*}(\alpha), \mathcal{R}_{1}(\alpha)=\mathcal{K}(\alpha)$ are well-known subclasses of $\mathcal{S}$ consisting of starlike functions of order $\alpha$ and convex functions of order $\alpha$ respectively. Let $\mathcal{T}, \mathcal{T S}^{*}(\alpha), \mathcal{T} \mathcal{K}(\alpha)$ and $\mathcal{T} \mathcal{R}_{\lambda}(\alpha)$ be respectively subclasses of $\mathcal{A}, \mathcal{S}^{*}(\alpha), \mathcal{K}(\alpha)$ and $\mathcal{R}_{\lambda}(\alpha)$ whose elements can be expressed in the form

$$
f(z)=z-\sum_{m=2}^{\infty}\left|a_{m}\right| z^{m}, \quad z \in \mathbb{D} .
$$


Om P. Ahuja, Subzar Beig and V. Ravichandran - Univalent Harmonic Functions

In [1], the first author observed that the family $\mathcal{R}_{\lambda}(\alpha)$ includes several other subclasses of $\mathcal{T}$. For example, the classes $\mathcal{R}[\alpha] \equiv \mathcal{R}_{1-2 \alpha}(\alpha)$ and $\mathcal{R}[\alpha, \beta] \equiv \mathcal{R}_{1-2 \alpha}(\beta)$ for $\alpha, \beta<1$ were respectively, studied in [17] and [4]. Recall that a function $f$ in $\mathcal{R}[\alpha]$ is called prestarlike of order $\alpha$ (see [3]).

Lemma 2. Let $f$ be an analytic function of the form (6), $\lambda \geq-1$ and $0 \leq \alpha<1$. Then the following statements are equivalent:

(1) $f \in \mathcal{T} \mathcal{R}_{\lambda}(\alpha)$

(2) $\left|\frac{z\left(\mathcal{D}^{\lambda+1} f(z)\right)}{\mathcal{D}^{\lambda} f(z)}-1\right| \leq 1-\alpha, \quad z \in \mathbb{D}$

(3) $\sum_{m=2}^{\infty} \frac{(m-\alpha)(\lambda+1)_{m-1}}{(1-\alpha)(m-1) !}\left|a_{m}\right| \leq 1$.

For $0 \leq \alpha<1$, let $\mathcal{F S}_{\mathcal{H}}^{*}(\alpha)$ and $\mathcal{F} \mathcal{K}_{\mathcal{H}}(\alpha)$ denote the subclasses of $\mathcal{H}$, respectively, consisting of fully starlike of order $\alpha$ and fully convex of order $\alpha$. These classes were studied in [12]. Recall that

$$
\begin{gathered}
\mathcal{F S}_{H}^{*}(\alpha)=\left\{f \in \mathcal{S}_{H}: \frac{\partial}{\partial \theta}\left(\arg \left(f\left(r e^{i \theta}\right)\right)\right) \geq \alpha, 0<r<1,0 \leq \theta<2 \pi\right\} \\
\mathcal{F K}_{\mathcal{H}}(\alpha)=\left\{f \in \mathcal{S}_{H}: \frac{\partial}{\partial \theta}\left(\arg \left(\frac{\partial}{\partial \theta} f\left(r e^{i \theta}\right)\right)\right) \geq \alpha, 0<r<1,0 \leq \theta<2 \pi\right\} .
\end{gathered}
$$

Let $\mathcal{T}_{H}, \mathcal{T} \mathcal{F S}_{H}^{*}(\alpha)$ and $\mathcal{T} \mathcal{F} \mathcal{K}_{H}(\alpha)$ be subclasses, respectively of $\mathcal{H}, \mathcal{F S}_{H}^{*}(\alpha)$ and $\mathcal{F} \mathcal{K}_{H}(\alpha)$ consisting of functions $f=h+\bar{g}$, where

$$
f(z)=z-\sum_{m=2}^{\infty}\left|a_{m}\right| z^{m}, \quad g(z)=\sum_{m=1}^{\infty}\left|b_{m}\right| z^{m}, \quad z \in \mathbb{D} .
$$

Lemma 3. [7] Let $\alpha \in[0,1)$ and the function $f=h+\bar{g}$ be given by (1). If the inequality

$$
\sum_{m=1}^{\infty}\left(\frac{m-\alpha}{1-\alpha}\left|a_{m}\right|+\frac{m+\alpha}{1-\alpha}\left|b_{m}\right|\right) \leq 2, \quad a_{1}=1,
$$

holds, then $f \in \mathcal{F S}_{H}^{*}(\alpha)$. However, if the function $f=h+\bar{g}$ is given by $(7)$, then the coefficient inequality (8) is necessary and sufficient for $f$ to be in $\mathcal{T F}_{H}^{*}(\alpha)$.

Lemma 4. [8] Let $\alpha \in[0,1)$ and the function $f=h+\bar{g}$ be given by (1). If the inequality

$$
\sum_{m=1}^{\infty}\left(\frac{m(m-\alpha)}{1-\alpha}\left|a_{m}\right|+\frac{m(m+\alpha)}{1-\alpha}\left|b_{m}\right|\right) \leq 2, \quad a_{1}=1,
$$

holds, then $f \in \mathcal{F K}_{H}(\alpha)$. However, if $f=h+\bar{g}$ is given by (7), then the coefficient inequality (9) is necessary and sufficient for $f$ to be in $\mathcal{T} \mathcal{F} \mathcal{K}_{H}(\alpha)$. 
Om P. Ahuja, Subzar Beig and V. Ravichandran - Univalent Harmonic Functions

If co-analytic part $g$ of the function $f=h+\bar{g}$ is zero, then Lemma 3 and Lemma 4 yield the following results.

Lemma 5. Let $\alpha \in[0,1)$ and the function $f \in \mathcal{T}$ be given by (6). Then

(a) $f \in \mathcal{T S}^{*}(\alpha) \Longleftrightarrow \sum_{m=2}^{\infty}(m-\alpha)\left|a_{m}\right| \leq 1-\alpha$,

(b) $f \in \mathcal{T} \mathcal{K}(\alpha) \Longleftrightarrow \sum_{m=2}^{\infty} m(m-\alpha)\left|a_{m}\right| \leq 1-\alpha$.

\section{MAIN RESUlts}

The first result of this section determines the condition for the local univalence of the operator $T_{\lambda, p}[f]$ defined by (2).

Lemma 6. Let $p>0, \lambda \geq 0$ and the function $f \in \mathcal{A}$. Then the function $T_{\lambda, p}[f]$ is locally univalent and sense-preserving in $\mathbb{D}$ if and only if $\mathcal{D}^{\lambda} f$ is convex in $\mathbb{D}$.

Proof. Write $T_{\lambda, p}[f]=H+\bar{G}$, where

$$
H=\frac{\mathcal{D}^{\lambda} f(z)+p z\left(\mathcal{D}^{\lambda} f(z)\right)^{\prime}}{p+1} \quad \text { and } \quad G=\frac{\mathcal{D}^{\lambda} f(z)-p z\left(\mathcal{D}^{\lambda} f(z)\right)^{\prime}}{p+1} .
$$

In view of Lewy's Theorem, $T_{\lambda, p}[f]$ is locally univalent and sense-preserving in $\mathbb{D}$ if and only if $\left|G^{\prime}\right|<\left|H^{\prime}\right|$, or equivalently if and only if

$$
\left|(1-p)\left(\mathcal{D}^{\lambda} f(z)\right)^{\prime}-p z\left(\mathcal{D}^{\lambda} f(z)\right)^{\prime \prime}\right|<\left|(1+p)\left(\mathcal{D}^{\lambda} f(z)\right)^{\prime}+p z\left(\mathcal{D}^{\lambda} f(z)\right)^{\prime \prime}\right| .
$$

Clearly $\left(D^{\lambda} f\right)^{\prime} \neq 0$ in $\mathbb{D}$, above inequality is equivalent to

$$
\left|\frac{1}{p}-\left(1+\frac{z\left(\mathcal{D}^{\lambda} f(z)\right)^{\prime \prime}}{\left(\mathcal{D}^{\lambda} f(z)\right)^{\prime}}\right)\right|<\left|\frac{1}{p}+\left(1+\frac{z\left(\mathcal{D}^{\lambda} f(z)\right)^{\prime \prime}}{\left(D^{\lambda} f(z)\right)^{\prime}}\right)\right|
$$

or

$$
\operatorname{Re}\left(1+\frac{z\left(\mathcal{D}^{\lambda} f(z)\right)^{\prime \prime}}{\left(\mathcal{D}^{\lambda} f(z)\right)^{\prime}}\right)>0
$$

This last condition is equivalent to convexity of $\mathcal{D}^{\lambda} f$.

For $\lambda=0$ and $p>0$, we have

Corollary 7. [10] For $f \in \mathcal{S}$, the function $T_{p}[f]$ defined in Example 1 is locally univalent and sense-preserving in $\mathbb{D}$ if and only if $f$ is convex analytic in $\mathbb{D}$. 
Om P. Ahuja, Subzar Beig and V. Ravichandran - Univalent Harmonic Functions

Corollary 8. Let $p>0, \lambda \geq 0$ and the function $f \in \mathcal{T}$ be given by (6). Then the function $T_{\lambda, p}[f]$ is locally univalent and sense-preserving in $\mathbb{D}$ if and only if

$$
\sum_{m=2}^{\infty} \frac{m^{2}(\lambda+1)_{m-1}}{(m-1) !}\left|a_{m}\right| \leq 1 .
$$

Proof. In view of Lemma $6, T_{\lambda, p}[f]$ is locally univalent and sense-preserving in $\mathbb{D}$ if and only if

$$
\left(\mathcal{D}^{\lambda} f\right)(z)=z-\sum_{m=2}^{\infty} \frac{(\lambda+1)_{m-1}}{(m-1) !}\left|a_{m}\right| z^{m},
$$

is convex. The result now follows from Lemma $5(\mathrm{~b})$.

Theorem 9. Let $p>0, \lambda \geq 0$ and the function $f \in \mathcal{A}$. Then the function $T_{\lambda, p}[f]$ is convex in the direction of imaginary axis if and only if $\mathcal{D}^{\lambda} f$ is convex in $\mathbb{D}$.

Proof. Suppose $T_{\lambda, p}[f]=H+\bar{G}$, where $H$ and $G$ are given by (10). The necessary part is obviously true by Lemma 6 . For the sufficient part, note that the analytic function

$$
M(z)=H(z)+G(z)=\frac{2 \mathcal{D}^{\lambda} f(z)}{p+1}
$$

satisfies

$$
\begin{gathered}
M^{\prime}=\frac{2}{p+1}\left(\mathcal{D}^{\lambda} f(z)\right)^{\prime} \neq 0 \\
\operatorname{Re}\left(1+\frac{z M^{\prime \prime}(z)}{M^{\prime}(z)}\right)=\operatorname{Re}\left(1+\frac{z\left(\mathcal{D}^{\lambda} f(z)\right)^{\prime \prime}}{\left(\mathcal{D}^{\lambda} f(z)\right)^{\prime}}\right)>0 .
\end{gathered}
$$

This, in particular, shows that $H+G$ is univalent and convex in the direction of imaginary axis. Using Theorem 1, we obtain the desired result.

Corollary 10. Let $p>0, \lambda \geq 0$ and the function $f \in \mathcal{T}$ be given by (6). Then the function $T_{\lambda, p}[f]$ is convex in the direction of imaginary axis if and only if the coefficient inequality

$$
\sum_{m=2}^{\infty} \frac{m^{2}(\lambda+1)_{m-1}}{(m-1) !}\left|a_{m}\right| \leq 1
$$

is satisfied.

Theorem 11. Suppose $0 \leq \alpha<1$ and $p>1$. Let the function $f \in \mathcal{A}$ is given by (3). If the condition

$$
\sum_{m=1}^{\infty} \frac{\left(p m^{2}-\alpha\right)(\lambda+1)_{m-1}}{(1-\alpha)(m-1) !(p+1)}\left|a_{m}\right| \leq 1, \quad a_{m}=1
$$


Om P. Ahuja, Subzar Beig and V. Ravichandran - Univalent Harmonic Functions

is satisfied, then the function $T_{\lambda, p}[f] \in \mathcal{F S}_{H}^{*}(\alpha)$ and the function $\mathcal{D}^{\lambda} f \in \mathcal{K}$. Moreover, if the function $f \in \mathcal{T}$ is given by (6), then (12) is necessary for the function $T_{\lambda, p}[f]$ to be in $\mathcal{T F S}_{H}^{*}(\alpha)$.

Proof. Using (2) and (3), we have

$$
T_{\lambda, p}[f](z)=\sum_{m=1}^{\infty} \frac{(1+p m)}{p+1} \frac{(\lambda+1)_{m-1}}{(m-1) !} a_{m} z^{m}+\sum_{m=1}^{\infty} \frac{\overline{(1-p m)}}{p+1} \frac{(\lambda+1)_{m-1}}{(m-1) !} a_{m} z^{m} .
$$

For $m \geq 1$, setting

$$
\mathcal{A}_{m}=\frac{(p m+1)(\lambda+1)_{m-1}}{(p+1)(m-1) !} a_{m}, \quad \text { and } \quad \mathcal{B}_{m}=\frac{(1-p m)(\lambda+1)_{m-1}}{(p+1)(m-1) !} a_{m}
$$

it can be seen that the inequality

$$
\sum_{m=1}^{\infty}\left(\frac{m-\alpha}{1-\alpha}\left|\mathcal{A}_{m}\right|+\frac{m+\alpha}{1-\alpha}\left|\mathcal{B}_{m}\right|\right) \leq 2,
$$

is equivalent to (12). By Lemma $3, T_{\lambda, p}[f] \in \mathcal{F S}_{H}^{*}(\alpha)$ and hence $\mathcal{D}^{\lambda} f \in \mathcal{K}$ by Lemma 6. In order to prove necessary condition, we assume that $a_{m} \leq 0$ for $m \geq 2$. It follows from (13) that $\mathcal{A}_{m} \leq 0$ for all $m \geq 2$ and $\mathcal{B}_{m} \geq 0$ for all $m \geq 1$. Again, it follows by Lemma 3 that (12) is satisfied if and only if $T_{\lambda, p}[f] \in \mathcal{T} \mathcal{F} \mathcal{S}_{H}^{*}(\alpha)$.

In [6], Goodman proved that if $f(z)=z+\sum_{m=2}^{\infty} a_{m} z^{m}$ is in $\mathcal{A}$ and if $\sum_{m=2}^{\infty} m^{2}\left|a_{m}\right| \leq$ 1 , then $f \in \mathcal{K}$. However, for $\alpha=0$, Theorem 11 provides the following stronger result.

Corollary 12. Under the hypothesis of Theorem 11, if the condition

$$
\sum_{m=2}^{\infty} \frac{m^{2}(\lambda+1)_{m-1}}{(m-1) !}\left|a_{m}\right| \leq \frac{1}{p}
$$

is satisfied, then the function $T_{\lambda, p}[f] \in \mathcal{F S}_{H}^{*}$ and the function $\mathcal{D}^{\lambda} f \in \mathcal{K}$.

Corollary 13. If the function $f(z)=z-\sum_{m=2}^{\infty} p\left|a_{m}\right| z^{m}, p \geq 1$ is in $\mathcal{T}$, then $\mathcal{D}^{\lambda} f \in \mathcal{T} \mathcal{K}$ if and only if $T_{\lambda, p}[f] \in \mathcal{T} \mathcal{F} \mathcal{S}_{H}^{*}$.

Proof. If $T_{\lambda, p}[f] \in \mathcal{T} \mathcal{F} \mathcal{S}_{H}^{*}$, then $T_{\lambda, p}[f]$ is locally univalent. By Lemma $6, \mathcal{D}^{\lambda} f \in$ $\mathcal{T} \mathcal{K}$. Conversely, suppose that $\mathcal{D}^{\lambda} f \in \mathcal{T} \mathcal{K}$. Note that

$$
\mathcal{D}^{\lambda} f(z)=z-\sum_{m=2}^{\infty} \frac{p(\lambda+1)_{m-1}}{(m-1) !}\left|a_{m}\right| z^{m}
$$

It follows from Lemma 5 that $D^{\lambda} f \in \mathcal{T} \mathcal{K}$ if and only if (14) holds. By Corollary 12, $T_{\lambda, p}[f] \in \mathcal{T} \mathcal{F} \mathcal{S}_{H}^{*}$. 
Om P. Ahuja, Subzar Beig and V. Ravichandran - Univalent Harmonic Functions

Theorem 14. Under the hypothesis of Theorem 11, if the condition

$$
\sum_{m=1}^{\infty} \frac{m\left(p m^{2}-\alpha\right)(\lambda+1)_{m-1}}{(1-\alpha)(p+1)(m-1) !}\left|a_{m}\right| \leq 1, \quad a_{1}=1,
$$

is satisfied, then $T_{\lambda, p}[f] \in \mathcal{F} \mathcal{K}_{H}(\alpha)$. Furthermore, if $a_{m} \leq 0$ for all $m \geq 2$, then the condition (15) is necessary for $T_{\lambda, p}[f]$ to be in $\mathcal{T} \mathcal{F} \mathcal{K}_{H}(\alpha)$.

Proof. Following the proof of Theorem 11, substituting $\mathcal{A}_{m}$ and $\mathcal{B}_{m}$ from (13), the inequality

$$
\sum_{m=1}^{\infty}\left(\frac{m(m-\alpha)}{1-\alpha}\left|\mathcal{A}_{m}\right|+\frac{m(m+\alpha)}{1-\alpha}\left|\mathcal{B}_{m}\right|\right) \leq 2,
$$

is equivalent to (15). By using Lemma 4 , it follows that $T_{\lambda, p}[f] \in \mathcal{F} \mathcal{K}_{H}(\alpha)$. On the other hand, if $a_{m} \leq 0$ for all $m \geq 2$, it is straight forward to see that $\mathcal{A}_{m} \leq 0$ for $m \geq 2$ and $\mathcal{B}_{m} \geq 0$ for $m \geq 1$. Thus $T_{\lambda, p}[f] \in \mathcal{T} \mathcal{F} \mathcal{K}_{H}(\alpha)$ if and only if (15) holds.

Theorem 15. Suppose $0 \leq \alpha<1$ and $p \geq 1$. If a function $f$ of the form (6) is in $\mathcal{T} \mathcal{R}_{\lambda}(\alpha)$, then

(a) $\left|\left(T_{\lambda, p}[f]\right)(z)\right| \leq \frac{2 p}{p+1} r+\frac{4 p(1-\alpha)}{(p+1)(2-\alpha)} r^{2}$,

(b) $\left|\left(T_{\lambda, p}[f]\right)(z)\right| \geq \frac{2 p}{p+1} r-\frac{4 p(1-\alpha)}{(p+1)(2-\alpha)} r^{2}$

where $|z| \mid=r<1$. The results are sharp.

Proof. Using (6) and (2), we obtain

$$
\begin{aligned}
\left|\left(T_{\lambda, p}[f]\right)(z)\right|=\left|z-\sum_{m=2}^{\infty} \frac{(p m+1)}{p+1} \frac{(\lambda+1)_{m-1}}{(m-1) !}\right| a_{m}\left|z^{m}\right| \\
\quad+\left|\frac{(1-p)}{p+1} z+\sum_{m=2}^{\infty} \frac{(1-p m)}{p+1} \frac{(\lambda+1)_{m-1}}{(m-1) !}\right| a_{m}\left|z^{m}\right| \\
\leq \frac{2 p}{p+1} r+\frac{2 p}{p+1}\left(\sum_{m=2}^{\infty} m \frac{(\lambda+1)_{m-1}}{(m-1) !}\left|a_{m}\right|\right) r^{2} \\
\leq \frac{2 p}{p+1} r+\frac{2 p(1-\alpha)}{p+1}\left(\sum_{m=2}^{\infty} \frac{(m-\alpha)(\lambda+1)_{m-1}}{(1-\alpha)(m-1) !}\left|a_{m}\right|\right) r^{2} \\
\quad+\frac{2 p(1-\alpha) \alpha}{(p+1)(2-\alpha)}\left(\sum_{m=2}^{\infty} \frac{(2-\alpha)(\lambda+1)_{m-1}}{(1-\alpha)(m-1) !}\left|a_{m}\right|\right) r^{2}
\end{aligned}
$$


Om P. Ahuja, Subzar Beig and V. Ravichandran - Univalent Harmonic Functions

$$
\begin{aligned}
& \leq \frac{2 p}{p+1} r+\frac{2 p(1-\alpha)}{p+1} r^{2} \\
& \quad+\frac{2 p(1-\alpha) \alpha}{(p+1)(2-\alpha)}\left(\sum_{m=2}^{\infty} \frac{(m-\alpha)(\lambda+1)_{m-1}}{(1-\alpha)(m-1) !}\left|a_{m}\right|\right) r^{2} \\
& \leq \frac{2 p}{p+1} r+\frac{4 p(1-\alpha)}{(p+1)(2-\alpha)} r^{2}
\end{aligned}
$$

by using Lemma 2 .

For the other inequality

$$
\begin{aligned}
\left|\left(T_{\lambda, p}[f]\right)(z)\right| \geq r-\sum_{m=2}^{\infty} \frac{(p m+1)}{p+1} \frac{(\lambda+1)_{m-1}}{(m-1) !}\left|a_{m}\right| r^{m} \\
\quad-\frac{(p-1)}{p+1} r-\sum_{m=2}^{\infty} \frac{(p m-1)(\lambda+1)_{m-1}}{(p+1)(m-1) !}\left|a_{m}\right| r^{m} \\
\geq \frac{2}{p+1} r-\frac{2 p}{p+1}\left(\sum_{m=2}^{\infty} \frac{\left.m(\lambda+1)_{m-1}\left|a_{m}\right|\right) r^{2}}{(m-1) !}\right. \\
=\frac{2}{p+1} r-\frac{2 p(1-\alpha)}{p+1}\left(\sum_{m=2}^{\infty} \frac{(m-\alpha)(\lambda+1)_{m-1}}{(1-\alpha)(m-1) !}\left|a_{m}\right|\right) r^{2} \\
\left.\geq \frac{2}{p+1} r-\frac{2 p(1-\alpha)}{p+1} r^{2} \frac{(2-\alpha)(\lambda+1)_{m-1}}{(p+1)(2-\alpha)}\left|a_{m}\right|\right) r^{2} \\
\quad-\frac{2 p(1-\alpha) \alpha}{(p+1)(2-\alpha)}\left(\sum_{m=2}^{\infty} \frac{(m-\alpha)(\lambda+1)_{m-1}}{(1-\alpha)(m-1) !}\left|a_{m}\right|\right) r^{2} \\
\geq \frac{2}{p+1} r-\frac{2 p(1-\alpha)}{p+1} r^{2}-\frac{2 p(1-\alpha) \alpha}{(p+1)(2-\alpha)} r^{2} \\
\geq \frac{2}{p+1} r-\frac{4 p(1-\alpha)}{(p+1)(2-\alpha)} r^{2} .
\end{aligned}
$$

\section{CONCLUDING REMARKS}

In this section, we introduce a new operator $T_{\lambda, p, \alpha}$ which is an extension of the operator $T_{\lambda, p}$. We will give some results as remarks which are nice extensions of some of the results in the previous section. 
Om P. Ahuja, Subzar Beig and V. Ravichandran - Univalent Harmonic Functions

For $f \in \mathcal{A}, \lambda \geq 0, p>0$ and $0 \leq \alpha<1$, we define for $z \in \mathbb{D}$,

$$
\begin{gathered}
T_{\lambda, p, \alpha}[f](z)=\frac{\mathcal{D}^{\lambda} f(z)+p\left(z\left(\mathcal{D}^{\lambda} f(z)\right)^{\prime}-\alpha \mathcal{D}^{\lambda} f(z)\right)}{1+p(1-\alpha)} \\
+\frac{\overline{\mathcal{D}^{\lambda} f(z)-p\left(z\left(\mathcal{D}^{\lambda} f(z)\right)^{\prime}-\alpha \mathcal{D}^{\lambda} f(z)\right)}}{1+p(1-\alpha)} .
\end{gathered}
$$

Clearly, we see that $T_{\lambda, p, 0}[f]=T_{\lambda, p}[f]$.

Remark 1. Going through the lines of the proof of Lemma 6, we see $T_{\lambda, p, \alpha}[f]$ is locally univalent and sense-preserving in $\mathbb{D}$ if and only if $\mathcal{D}^{\lambda} f$ is convex of order $\alpha$ in $\mathbb{D}$ for all $p>0$ and $\lambda \geq 0$.

Remark 2. Suppose $f(z)=z-\sum_{m=2}^{\infty}\left|a_{m}\right| z^{m} \in \mathcal{T}$. Then the operator $T_{\lambda, p, \alpha}[f]$ is locally univalent and sense-preserving in $\mathbb{D}$ if and only if

$$
\sum_{m=2}^{\infty} \frac{m(m-\alpha)(\lambda+1)_{m-1}}{(m-1) !}\left|a_{m}\right| \leq(1-\alpha), \quad p>0, \quad \lambda \geq 0, \quad 0 \leq \alpha<1 .
$$

Proof. In view of Remark $1, T_{\lambda, p, \alpha}[f]$ is locally univalent and sense-preserving in $\mathbb{D}$ if and only if

$$
\left(\mathcal{D}^{\lambda} f\right)(z)=z-\sum_{m=2}^{\infty} \frac{(\lambda+1)_{m-1}}{(m-1) !}\left|a_{m}\right| z^{m}
$$

is convex of order $\alpha$. The result now follows from Lemma 5(b).

The reader can also check the corresponding results regarding the operator $T_{\lambda, p, \alpha}$ which are done for $T_{\lambda, p}$ in the previous section.

\section{REFERENCES}

[1] O. P. Ahuja, Hadamard products of analytic functions defined by Ruscheweyh derivatives, in Current topics in analytic function theory, 13-28, World Sci. Publ., River Edge, NJ, 1992.

[2] O. P. Ahuja and M. Nunokawa, Neighborhoods of analytic functions defined by Ruscheweyh derivatives, Math. Japon. 51 (2000), no. 3, 487-492.

[3] O. P. Ahuja and H. Silverman, Convolutions of prestarlike functions, Internat. J. Math. Math. Sci. 6 (1983), no. 1, 59-68.

[4] H. S. Al-Amiri, Certain generalizations of prestarlike functions, J. Austral. Math. Soc. Ser. A 28 (1979), no. 3, 325-334. 
Om P. Ahuja, Subzar Beig and V. Ravichandran - Univalent Harmonic Functions

[5] J. Clunie and T. Sheil-Small, Harmonic univalent functions, Ann. Acad. Sci. Fenn. Ser. A I Math. 9 (1984), 3-25.

[6] Goodman, A. W. Univalent functions and nonanalytic curves. Proc. Amer. Math. Soc. 8 (1957), 598-601.

[7] J. M. Jahangiri, Harmonic functions starlike in the unit disk, J. Math. Anal. Appl. 235 (1999), no. 2, 470-477.

[8] J. M. Jahangiri, Coefficient bounds and univalence criteria for harmonic functions with negative coefficients, Ann. Univ. Mariae Curie-Skłodowska Sect. A 52 (1998), no. 2, 57-66.

[9] H. Lewy, On the non-vanishing of the Jacobian in certain one-to-one mappings, Bull. Amer. Math. Soc. 42 (1936), no. 10, 689-692.

[10] S. Muir, Weak subordination for convex univalent harmonic functions, J. Math. Anal. Appl. 348 (2008), no. 2, 862-871.

[11] S. Muir, Harmonic mappings convex in one or every direction, Comput. Methods Funct. Theory 12 (2012), no. 1, 221-239.

[12] S. Nagpal and V. Ravichandran, Fully starlike and fully convex harmonic mappings of order $\alpha$, Ann. Polon. Math. 108 (2013), no. 1, 85-107.

[13] S. Ruscheweyh, New criteria for univalent functions, Proc. Amer. Math. Soc. 49 (1975), 109-115.

[14] S. Ruscheweyh and L. C. Salinas, On the preservation of direction-convexity and the Goodman-Saff conjecture, Ann. Acad. Sci. Fenn. Ser. A I Math. 14 (1989), no. $1,63-73$.

[15] S. Ruscheweyh and T. J. Suffridge, A continuous extension of the de la Vallée Poussin means, J. Anal. Math. 89 (2003), 155-167.

[16] T. Sheil-Small, Constants for planar harmonic mappings, J. London Math. Soc. (2) 42 (1990), no. 2, 237-248.

[17] H. Silverman and E. M. Silvia, Prestarlike functions with negative coefficients, Internat. J. Math. Math. Sci. 2 (1979), no. 3, 427-439.

Om P. Ahuja

Department of Mathematics

Kent State University

Burton, USA

email: oahuja@kent.edu

Subzar Beig

Department of Mathematics 
Om P. Ahuja, Subzar Beig and V. Ravichandran - Univalent Harmonic Functions

University of Delhi

Delhi-110 007, India

email: beighsubzar@gmail.com

V. Ravichandran

Department of Mathematics

University of Delhi

Delhi-110007, India

email:vravi@maths.du.ac.in; vravi68@gmail.com 